\title{
Twisted Deformation Quantization of Algebraic Varieties (Survey)
}

\author{
Amnon Yekutieli
}

\begin{abstract}
Let $X$ be a smooth algebraic variety over a field of characteristic 0 . We introduce the notion of twisted associative (resp. Poisson) deformation of the structure sheaf $\mathcal{O}_{X}$. These are stack-like versions of usual deformations. We prove that there is a twisted quantization map from twisted Poisson deformations to twisted associative deformations, which is canonical and bijective on equivalence classes.
\end{abstract}

\section{Introduction}

Let $\mathbb{K}$ be a field of characteristic 0 , and let $X$ be a smooth algebraic variety over $\mathbb{K}$, with structure sheaf $\mathcal{O}_{X}$. An associative deformation of $\mathcal{O}_{X}$ is a sheaf $\mathcal{A}$ of flat complete associative $\mathbb{K}[[\hbar]]$-algebras on $X$, with an isomorphism $\mathbb{K} \otimes_{\mathbb{K}[[\hbar]]} \mathcal{A} \cong \mathcal{O}_{X}$, called an augmentation. Similarly, a Poisson deformation of $\mathcal{O}_{X}$ is a sheaf $\mathcal{A}$ of flat complete commutative Poisson $\mathbb{K}[[\hbar]]$-algebras on $X$, with an augmentation to $\mathcal{O}_{X}$.

In this paper we introduce the notion of twisted associative (resp. Poisson) deformation of $\mathcal{O}_{X}$. A twisted deformation (or either kind) is a stack-like version of an ordinary deformation. The precise definition is given in Section 5 But to give an idea, let us say that a twisted deformation $\mathcal{A}$ can be described as a collection of locally defined deformations $\mathcal{A}_{i}$, each living on an open set $U_{i}$ of $X$, that are glued together in a loose way. We should also say that an associative deformation $\mathcal{A}$ is a special kind of stack of algebroids, in the sense of Ko2. Indeed, one reason for introducing twisted deformations is to have a Poisson analogue of a stack of algebroids.

There is a notion of twisted gauge equivalence between twisted associative (resp. Poisson) deformation of $\mathcal{O}_{X}$. A twisted deformation $\mathcal{A}$ induces a first order bracket $\{-,-\}_{\mathcal{A}}$ on $\mathcal{O}_{X}$. The main result is Theorem 6.1, which says that there is a

Key words and phrases. Deformation quantization, algebraic varieties, stacks, gerbes, DG Lie algebras.

Mathematics Subject Classification 2000. Primary: 53D55; Secondary: 14B10, 16S80, 17B40, $18 \mathrm{D} 05$.

This research was supported by the US-Israel Binational Science Foundation and by the Israel Science Foundation.

(c) 0000 (copyright holder) 
canonical bijection of sets

$$
\text { tw.quant } \begin{aligned}
: & \frac{\left\{\text { twisted Poisson deformations of } \mathcal{O}_{X}\right\}}{\text { twisted gauge equivalence }} \\
& \stackrel{\simeq}{\longrightarrow} \frac{\left\{\text { twisted associative deformations of } \mathcal{O}_{X}\right\}}{\text { twisted gauge equivalence }}
\end{aligned}
$$

called the twisted quantization map. It preserves first order brackets, and commutes with étale morphisms $X^{\prime} \rightarrow X$.

This survey article is an edited version of lectures that I gave on several occasions. In addition to the main body of the article, there are four appendices, that provide further details on certain aspects of the topic. Full details can be found in my papers listed in the bibliography. The bibliography also covers work by other researchers in this area.

Acknowledgments. Part of the work presented here is joint with Frederick Leitner. Many of the ideas in this paper are influenced by the work of Maxim Kontsevich, and I wish to thank him for discussing this material with me. Thanks also to Michael Artin, Pavel Etingof, Damien Calaque, Michel Van den Bergh, Pierre Deligne, Lawrence Breen, Pierre Schapira and James Stasheff for their assistance on various aspects of the paper.

\section{Some background on Deformation Quantization}

Throughout $\mathbb{K}$ is a field of characteristic 0 .

Let $C$ be a commutative $\mathbb{K}$-algebra. Recall that a Poisson bracket on $C$ is a $\mathbb{K}$-bilinear function

$$
\{-,-\}: C \times C \rightarrow C
$$

which makes $C$ into a Lie algebra, and is a biderivation (i.e. a derivation in each argument). The pair $(C,\{-,-\})$ is called a Poisson algebra.

Poisson algebras arise in several ways, e.g. classical Hamiltonian mechanics, or Lie theory.

Let $\mathbb{K}[[\hbar]]$ be the ring of formal power series in the variable $\hbar$. And let $C[[\hbar]]$ be the set of formal power series with coefficients in $C$, which we view only as a $\mathbb{K}[[\hbar]]$-module. A star product on $C[[\hbar]]$ is a $\mathbb{K}[[\hbar]]$-bilinear function

$$
\star: C[[\hbar]] \times C[[\hbar]] \rightarrow C[[\hbar]]
$$

which makes $C[[\hbar]]$ into an associative $\mathbb{K}[[\hbar]]$-algebra, with unit $1 \in C$, and such that

$$
f \star g \equiv f g \bmod \hbar
$$

for any $g, f \in C$.

Note that the star product $\star$ can be expanded into a power series as follows: there is a sequence $\left\{\omega_{j}\right\}_{j \geq 1}$ of $\mathbb{K}$-bilinear functions $\omega_{j}: C \times C \rightarrow C$, such that

$$
f \star g:=f g+\sum_{j=1}^{\infty} \omega_{j}(f, g) \hbar^{j}
$$

for $f, g \in C$. The conditions that $\star$ is associative and unital place certain constraints on the sequence $\left\{\omega_{j}\right\}_{j \geq 1}$. 
Example 1.2. Suppose $\star$ is a star product on $C[[\hbar]]$. Given $f, g \in C$, we know that

$$
f \star g-g \star f \equiv 0 \bmod \hbar .
$$

Hence there is a unique element $\{f, g\}_{\star} \in C$ such that

$$
\frac{1}{2 \hbar}(f \star g-g \star f) \equiv\{f, g\}_{\star} \bmod \hbar .
$$

It is quite easy to show that $\{-,-\}_{\star}$ is a Poisson bracket on $C$. We call it the first order bracket of $\star$.

Deformation quantization seeks to reverse Example 1.2 .

Definition 1.3. Given a Poisson bracket $\{-,-\}$ on the algebra $C$, a deformation quantization of $\{-,-\}$ is a star product $\star$ on $C[[\hbar]]$ whose first order bracket is $\{-,-\}$.

In physics $\hbar$ is the Planck constant. For a quantum phenomenon depending on $\hbar$, the limit as $\hbar \rightarrow 0$ is thought of the as the classical limit of this phenomenon.

The original idea by the physicists Flato et. al. ([BFFLS], 1978) was that deformation quantization should model the transition from classical Hamiltonian mechanics to quantum mechanics. Special cases (like the Moyal product) were known. The problem arose: does any Poisson bracket admit a deformation quantization?

For a symplectic manifold $X$ and $C=\mathrm{C}^{\infty}(X)$ the problem was solved by De Wilde and Lecomte $([\mathbf{D L}, 1983)$. A more geometric solution was discovered by Fedosov $\left([\mathbf{F e}, 1994)\right.$. The general case, i.e. $C=\mathrm{C}^{\infty}(X)$ for a Poisson manifold $X$, was solved by Kontsevich ([Ko1, 1997). See surveys in the book [CKTB].

Remark 1.4. The deformations considered in this paper are parameterized by the algebra $\mathbb{K}[[\hbar]]$. It is possible to replace $\mathbb{K}[[\hbar]]$ with any other noetherian complete local commutative $\mathbb{K}$-algebra $R$, with maximal ideal $\mathfrak{m}$, such that $R / \mathfrak{m}=\mathbb{K}$. Instead of $C[[\hbar]]$ we take the complete tensor product $R \widehat{\otimes}_{\mathbb{K}} C$, with its obvious augmentation to $C$. All results will remain valid.

\section{Poisson Deformations of Algebraic Varieties}

In algebraic geometry we have to consider deformations as sheaves.

Let $X$ be a smooth algebraic variety over $\mathbb{K}$, with structure sheaf $\mathcal{O}_{X}$. We view $\mathcal{O}_{X}$ as a Poisson $\mathbb{K}$-algebra with zero bracket.

Definition 2.1. A Poisson deformation of $\mathcal{O}_{X}$ is a sheaf $\mathcal{A}$ of flat, $\hbar$-adically complete, commutative Poisson $\mathbb{K}[[\hbar]]$-algebras on $X$, with an isomorphism of Poisson algebras

called an augmentation.

$$
\psi: \mathcal{A} /(\hbar) \stackrel{\simeq}{\longrightarrow} \mathcal{O}_{X}
$$

A gauge equivalence $\mathcal{A} \rightarrow \mathcal{A}^{\prime}$ between Poisson deformations is a $\mathbb{K}[[\hbar]]$-linear isomorphism of sheaves of Poisson algebras, that commutes with the augmentations to $\mathcal{O}_{X}$.

It may happen that $\mathcal{A} \cong \mathcal{O}_{X}[[\hbar]]$ as sheaves of commutative $\mathbb{K}[[\hbar]]$-algebras augmented to $\mathcal{O}_{X}$; if this is so, then we say that $\mathcal{A}$ is a sheaf-theoretically trivial deformation. A sufficient condition for that is the vanishing of the cohomology group $\mathrm{H}^{1}\left(X, \mathcal{T}_{X}\right)$, where $\mathcal{T}_{X}$ is the tangent sheaf. The corresponding $\mathbb{K}[[\hbar]]$-bilinear Poisson bracket on $\mathcal{O}_{X}[[\hbar]]$ is called a formal Poisson bracket. 
Given a Poisson deformation $\mathcal{A}$ of $\mathcal{O}_{X}$, we may define the first order bracket

$$
\{-,-\}_{\mathcal{A}}: \mathcal{O}_{X} \times \mathcal{O}_{X} \rightarrow \mathcal{O}_{X} \text {. }
$$

This is a Poisson bracket whose formula is

$$
\{f, g\}_{\mathcal{A}}:=\psi\left(\frac{1}{\hbar}\{\tilde{f}, \tilde{g}\}\right),
$$

where $f, g \in \mathcal{O}_{X}$ are local sections, and $\tilde{f}, \tilde{g} \in \mathcal{A}$ are arbitrary local lifts. The first order bracket is invariant under gauge equivalences.

Example 2.2. Let $\{-,-\}_{1}$ be some Poisson bracket on $\mathcal{O}_{X}$. Put on the commutative $\mathbb{K}[[\hbar]]$-algebra $\mathcal{A}:=\mathcal{O}_{X}[[\hbar]]$ the formal Poisson bracket $\hbar\{-,-\}_{1}$, namely

$$
\{f, g\}=\hbar\{f, g\}_{1}
$$

for $f, g \in \mathcal{O}_{X}$. Then $\mathcal{A}$ is a Poisson deformation of $\mathcal{O}_{X}$. The first order bracket in this case is just

$$
\{-,-\}_{\mathcal{A}}=\{-,-\}_{1} .
$$

Poisson deformations are controlled by a coherent sheaf of DG (differential graded) Lie algebras $\mathcal{T}_{\text {poly }, X}$, called the poly derivations. This is explained in Appendix A.

\section{Associative Deformations of Algebraic Varieties}

Let $X$ be a smooth algebraic variety over $\mathbb{K}$ as before.

Definition 3.1. An associative deformation of $\mathcal{O}_{X}$ is a sheaf $\mathcal{A}$ of flat, $\hbar$-adically complete, associative, unital $\mathbb{K}[[\hbar]]$-algebras on $X$, with an isomorphism of algebras

$$
\psi: \mathcal{A} /(\hbar) \stackrel{\simeq}{\longrightarrow} \mathcal{O}_{X}
$$

called an augmentation.

A gauge equivalence $\mathcal{A} \rightarrow \mathcal{A}^{\prime}$ between associative deformations is a $\mathbb{K}[[\hbar]]$-linear isomorphism of sheaves of unital algebras, that commutes with the augmentations to $\mathcal{O}_{X}$.

It may happen that $\mathcal{A} \cong \mathcal{O}_{X}[[\hbar]]$ as sheaves of $\mathbb{K}[[\hbar]]$-modules augmented to $\mathcal{O}_{X}$; if this is so, then we say that $\mathcal{A}$ is a sheaf-theoretically trivial deformation. A sufficient condition for that is the vanishing of the cohomology group $\mathrm{H}^{1}\left(X, \mathcal{D}_{X}\right)$, where $\mathcal{D}_{X}$ is the sheaf of differential operators on $X$. (This fact is quite hard to prove, and it relies on Theorem A.5) The corresponding $\mathbb{K}[[\hbar]]$-bilinear multiplication on $\mathcal{O}_{X}[[\hbar]]$ is called a star product, like in Section 1.

Given an associative deformation $\mathcal{A}$ we may define the first order bracket

$$
\{-,-\}_{\mathcal{A}}: \mathcal{O}_{X} \times \mathcal{O}_{X} \rightarrow \mathcal{O}_{X} .
$$

The formula, in terms of local sections, is

$$
\{f, g\}_{\mathcal{A}}:=\psi\left(\frac{1}{2 \hbar}(\tilde{f} \star \tilde{g}-\tilde{g} \star \tilde{f})\right) .
$$

The first order bracket is invariant under gauge equivalences.

Associative deformations are controlled by a quasi-coherent sheaf of DG Lie algebras $\mathcal{D}_{\text {poly }, X}$, called the poly differential operators. This is explained in Appendix A.

Note that both kinds of deformations - Poisson and associative - include as special cases the classical commutative deformations of $\mathcal{O}_{X}$. 


\section{Deformation Quantization}

Kontsevich Ko1 proved that any Poisson deformation of a real $\mathrm{C}^{\infty}$ manifold $X$ can be canonically quantized. In this section we present an algebraic version of this result. But first a definition.

Definition 4.1. Let $\mathcal{A}$ be a Poisson deformation of $\mathcal{O}_{X}$. A quantization of $\mathcal{A}$ is an associative deformation $\mathcal{B}$, such that the first order brackets satisfy

$$
\{-,-\}_{\mathcal{B}}=\{-,-\}_{\mathcal{A}} \text {. }
$$

Recalling Example 2.2, we see that this definition captures the essence of deformation quantization, namely quantizing a Poisson bracket on $\mathcal{O}_{X}$.

Theorem 4.2 ([Ye1]). Let $\mathbb{K}$ be a field containing $\mathbb{R}$, and let $X$ be a smooth affine algebraic variety over $\mathbb{K}$. There is a canonical bijection

$$
\text { quant : } \frac{\left\{\text { formal Poisson brackets on } \mathcal{O}_{X}[[\hbar]]\right\}}{\text { gauge equivalence }} \stackrel{\simeq}{\longrightarrow} \frac{\left\{\text { star products on } \mathcal{O}_{X}[[\hbar]]\right\}}{\text { gauge equivalence }} \text {. }
$$

which is a quantization as defined above.

By "canonical" we mean that this quantization map commutes with étale morphisms $X^{\prime} \rightarrow X$ (and in particular with automorphisms of $X$ ).

Actually our result in [Ye1 is stronger - it holds for a wider class of varieties, not just affine varieties. However all these cases are subsumed in Corollary 6.3 below.

On the other hand, it might be good to remark that when writing Ye1 we did not know Theorem A.5 and hence we only considered differential star products in that paper. Now we know that there is no difference (up to gauge equivalence), so Theorem 4.2 is correct as stated. In the context of complex manifolds this issue is still open: it is not known if every star product is gauge equivalent to a differential one! See $\mathbf{K S 2}$.

It is important to note that even the affine case of Theorem 4.2 is a "global result". In this context "local" means a sufficiently small affine open set $U \subset X$ that admits an étale coordinate system, namely an étale morphism $U \rightarrow \mathbf{A}_{\mathbb{K}}^{n}$. This is totally analogous to the case of $\mathrm{C}^{\infty}$ manifolds studied by Kontsevich, where "local" meant an open set in the manifold $\mathbf{A}_{\mathbb{R}}^{n}=\mathbb{R}^{n}$.

Theorem 4.2 is a consequence of the following more general result.

Theorem 4.3 (鸟1). Let $\mathbb{K}$ be a field containing $\mathbb{R}$, and let $X$ be a smooth algebraic variety over $\mathbb{K}$. There is a diagram

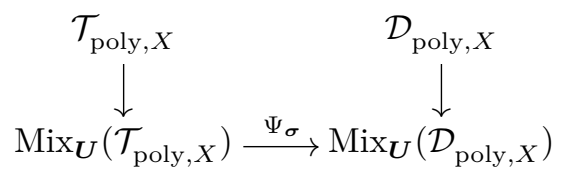

where:

- $\operatorname{Mix}_{U}\left(\mathcal{T}_{\text {poly }, X}\right)$ and $\operatorname{Mix}_{U}\left(\mathcal{D}_{\text {poly }, X}\right)$ are sheaves of DG Lie algebras on $X$, called mixed resolutions;

- the vertical arrows are DG Lie algebra quasi-isomorphisms;

- and the horizontal arrow $\Psi_{\sigma}$ is an $\mathrm{L}_{\infty}$ quasi-isomorphism. 
The mixed resolutions combine the commutative Čech resolution associated to a sufficiently refined affine open covering $\boldsymbol{U}$ of $X$, and the Grothendieck sheaf of jets. An $\mathrm{L}_{\infty}$ quasi-isomorphism is a generalization of a DG Lie algebra quasiisomorphism. The $\mathrm{L}_{\infty}$ quasi-isomorphism $\Psi_{\boldsymbol{\sigma}}$ depends on some choices; but the dependence on these choices and on the covering $\boldsymbol{U}$ disappears in homotopy. Theorem 4.3 is proved using the Formality Theorem of Kontsevich [Ko1] and formal geometry. More on the proof of Theorem 4.3 in Appendices B and C. A somewhat different approach to Theorem 4.3 can be found in Van den Bergh's paper [VdB].

\section{Twisted Deformations of Algebraic Varieties}

What can be done in general, when the variety $X$ is not affine? Can we still make use of Theorem 4.3.

In the paper Ko3 Kontsevich suggests that in general the deformation quantization of a Poisson bracket might have to be a stack of algebroids. This is a generalization of the notion of sheaf of algebras.

Actually stacks of algebroids appeared earlier, under the name sheaves of

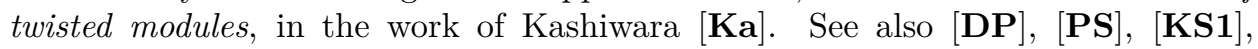
KS2.

I will use the term twisted associative deformation, and present an approach that treats the Poisson case as well. A similar point of view is taken in [BGNT].

Here I will explain only a naive definition of twisted deformations. A more sophisticated definition, involving gerbes, may be found in Appendix D. The fact that the two definitions agree follows from our work on central extensions of gerbes and obstructions classes [Ye5].

Let $U \subset X$ be an affine open set, and let $C:=\Gamma\left(U, \mathcal{O}_{X}\right)$. Suppose $A$ is an associative or Poisson deformation of the $\mathbb{K}$-algebra $C$. One may assume that $A=C[[\hbar]]$, and it is either endowed with a Poisson bracket $\{-,-\}$, or with a star product $\star$.

In either case $A$ becomes a pronilpotent Lie algebra, and $\hbar A$ is a Lie subalgebra. In the Poisson case the Lie bracket is $\{-,-\}$, and in the associative case the Lie bracket is the commutator

$$
[a, b]:=a \star b-b \star a .
$$

Let us denote the corresponding pronilpotent group by

$$
\operatorname{IG}(A):=\exp (\hbar A)
$$

and call it the group of inner gauge transformations of $A$.

The group $\operatorname{IG}(A)$ acts on the deformation $A$ by gauge equivalences. We denote this action by ig. In the Poisson case the gauge transformation $\operatorname{ig}(g)$, for $g \in \operatorname{IG}(A)$, can be viewed as a formal hamiltonian flow. In the associative case the intrinsic exponential function

$$
\exp (a)=\sum_{i \geq 0} \frac{1}{i !} \underbrace{a \star \cdots \star a}_{i},
$$

for $a \in \hbar A$, allows us to identify the group $\operatorname{IG}(A)$ with the multiplicative subgroup

$$
\{g \in A \mid g \equiv 1 \bmod \hbar\} .
$$

Under this identification the operation $\operatorname{ig}(g)$ is just conjugation by the invertible element $g$. 
The above can be sheafified: to a deformation $\mathcal{A}$ of $\mathcal{O}_{X}$ we assign the sheaf of groups $\operatorname{IG}(\mathcal{A})$, etc.

Let us fix an affine open covering $\left\{U_{0}, \ldots, U_{m}\right\}$ of $X$. We write

$$
U_{i, j, \ldots}:=U_{i} \cap U_{j} \cap \cdots .
$$

Definition 5.1. A twisted associative (resp. Poisson) deformation $\mathcal{A}$ of $\mathcal{O}_{X}$ consists of the following data:

(1) For any $i$, a deformation $\mathcal{A}_{i}$ of $\mathcal{O}_{U_{i}}$.

(2) For any $i<j$, a gauge equivalence

$$
g_{i, j}:\left.\left.\mathcal{A}_{i}\right|_{U_{i, j}} \rightarrow \mathcal{A}_{j}\right|_{U_{i, j}} .
$$

(3) For any $i<j<k$, an element

$$
a_{i, j, k} \in \Gamma\left(U_{i, j, k}, \operatorname{IG}\left(\mathcal{A}_{i}\right)\right) .
$$

The conditions are:

(i) For any $i<j<k$ one has

$$
g_{i, k}^{-1} \circ g_{j, k} \circ g_{i, j}=\operatorname{ig}\left(a_{i, j, k}^{-1}\right) .
$$

(ii) For any $i<j<k<l$ one has

$$
a_{i, j, l}^{-1} \cdot a_{i, k, l} \cdot a_{i, j, k}=g_{i, j}^{-1}\left(a_{j, k, l}\right) .
$$

Condition (i) says that the 2-cochain $\left\{a_{i, j, k}\right\}$ measures the failure of the 1cochain $\left\{g_{i, j}\right\}$ to be a cocycle. This tells us whether the collection $\left\{\mathcal{A}_{i}\right\}$ of local deformations can be glued into a global deformation of $\mathcal{O}_{X}$.

Condition (ii) - usually called the tetrahedron equation - says that the 2cochain $\left\{a_{i, j, k}\right\}$ satisfies a twisted cocycle condition.

See Figure 1 for an illustration.

Example 5.2. If $\mathcal{A}$ is a usual deformation of $\mathcal{O}_{X}$, then we obtain a twisted deformation $\mathcal{A}$ by taking $\mathcal{A}_{i}:=\left.\mathcal{A}\right|_{U_{i}}, g_{i, j}:=\mathbf{1}$ and $a_{i, j, k}:=1$.

In this way we can view usual deformations as twisted deformations.

There is a notion of twisted gauge equivalence $\mathcal{A} \rightarrow \mathcal{A}^{\prime}$ between twisted associative (resp. Poisson) deformations of $\mathcal{O}_{X}$.

Let $\mathcal{A}$ be a twisted deformation. We say $\mathcal{A}$ is really twisted if it is not twistedequivalent to any usual deformation $\mathcal{A}^{\prime}$.

Example 5.3. It is easy to construct an example of a commutative twisted associative deformation $\mathcal{A}$ of $\mathcal{O}_{X}$ that is really twisted. Take an algebraic variety $X$ with nonzero cohomology class $c \in \mathrm{H}^{2}\left(X, \mathcal{O}_{X}\right)$. Let $\boldsymbol{U}$ be an affine open covering of $X$, and let $\left\{c_{i, j, k}\right\}$ be a Čech 2-cocycle representing $c$ on this covering. Now consider the data $\left(\left\{\mathcal{A}_{i}\right\},\left\{g_{i, j}\right\},\left\{a_{i, j, k}\right\}\right)$ with $\mathcal{A}_{i}:=\mathcal{O}_{U_{i}}[[\hbar]], g_{i, j}:=\mathbf{1}$ and

$$
a_{i, j, k}:=\exp \left(\hbar c_{i, j, k}\right) .
$$

This twisted deformation $\mathcal{A}$ has obstruction class $c$ in the first order central extension (in the sense of [Ye6]). This implies that $\mathcal{A}$ is really twisted.

Remark 5.4. For a twisted associative deformation $\mathcal{A}$ there is a well defined abelian category $\operatorname{Coh} \mathcal{A}$ of "coherent left $\mathcal{A}$-modules", which is a deformation of the abelian category $\operatorname{Coh} \mathcal{O}_{X}$. See the work of Lowen and Van den Bergh $[\mathbf{L V}]$. 


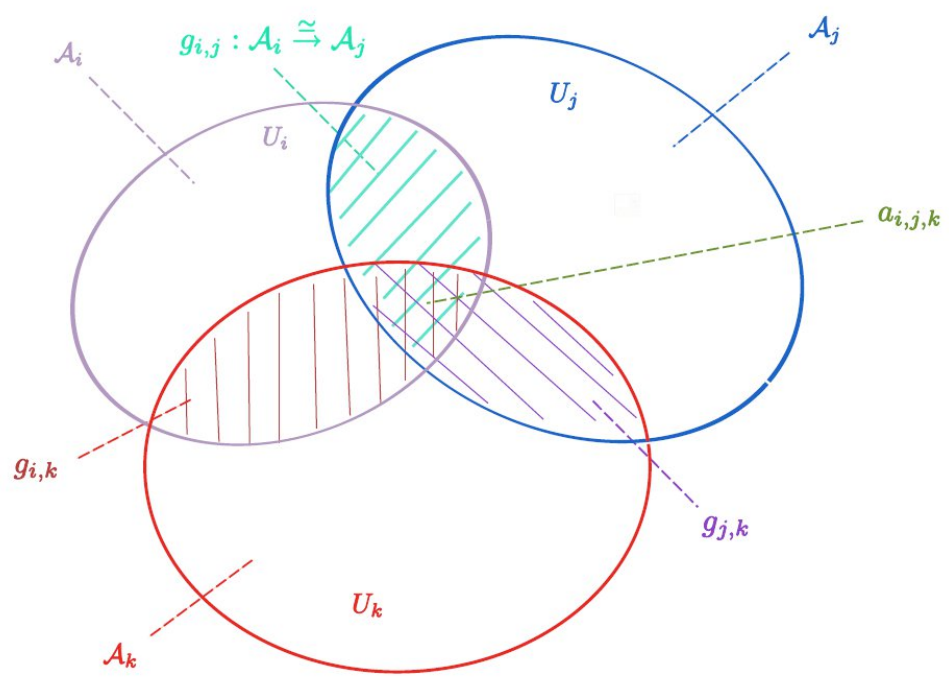

FiguRE 1.

Indeed, there is a geometric Morita theory, which says that twisted associative deformations of $\mathcal{O}_{X}$ are the same as deformations of $\operatorname{Coh} \mathcal{O}_{X}$. This is explained in the last chapter of the book [KS1] by Kashiwara and Schapira.

We do not know of a similar interpretation of twisted Poisson deformations.

\section{Twisted Deformation Quantization}

Just as in the case of usual deformations, given a twisted (associative or Poisson) deformation $\mathcal{A}$ of $\mathcal{O}_{X}$, we can define the first order bracket $\{-,-\}_{\mathcal{A}}$ on $\mathcal{O}_{X}$. The first order bracket is invariant under twisted gauge equivalence.

Let $\mathcal{A}$ be a twisted Poisson deformation, and let $\mathcal{B}$ be a twisted associative deformation. We say that $\mathcal{B}$ is a twisted quantization of $\mathcal{A}$ if

$$
\{-,-\}_{\mathcal{B}}=\{-,-\}_{\mathcal{A}} .
$$

The next theorem is influenced by ideas of Kontsevich (from [Ko3] and private communications).

Theorem 6.1 ([Y Ye5]). Let $\mathbb{K}$ be a field containing $\mathbb{R}$, and let $X$ be a smooth algebraic variety over $\mathbb{K}$. There is a canonical bijection

$$
\text { tw.quant } \begin{aligned}
& \frac{\left\{\text { twisted Poisson deformations of } \mathcal{O}_{X}\right\}}{\text { twisted gauge equivalence }} \\
& \stackrel{\simeq}{\longrightarrow} \frac{\left\{\text { twisted associative deformations of } \mathcal{O}_{X}\right\}}{\text { twisted gauge equivalence }},
\end{aligned}
$$

called the twisted quantization map, which is a twisted quantization in the sense above. 
As before, by "canonical" we mean that the twisted quantization map commutes with étale morphisms $X^{\prime} \rightarrow X$.

The proof of Theorem 6.1 relies on a rather complicated calculations involving Maurer-Cartan equations in cosimplicial DG Lie algebras, and cosimplicial 2groupoids. See Appendices C and D.

The results on pronilpotent gerbes from [Ye6] tell us that when $\mathrm{H}^{1}\left(X, \mathcal{O}_{X}\right)=0$ and $\mathrm{H}^{2}\left(X, \mathcal{O}_{X}\right)=0$, there are no really twisted deformations. Thus Theorem 6.1 implies:

Corollary 6.2. Assume the cohomology groups $\mathrm{H}^{1}\left(X, \mathcal{O}_{X}\right)$ and $\mathrm{H}^{2}\left(X, \mathcal{O}_{X}\right)$ vanish. Then the function tw.quant of the theorem gives a bijection

$$
\text { quant : } \frac{\left\{\text { Poisson deformations of } \mathcal{O}_{X}\right\}}{\text { gauge equivalence }} \stackrel{\text { as }}{\longrightarrow} \frac{\left\{\text { associative deformations of } \mathcal{O}_{X}\right\}}{\text { gauge equivalence }} \text {. }
$$

As we already mentioned, if $\mathrm{H}^{1}\left(X, \mathcal{T}_{X}\right)=0$, then all twisted Poisson deformations are sheaf theoretically trivial. Likewise, if $\mathrm{H}^{1}\left(X, \mathcal{D}_{X}\right)=0$, then all twisted associative deformations are sheaf theoretically trivial. Hence:

Corollary 6.3. Assume the cohomology groups $\mathrm{H}^{1}\left(X, \mathcal{O}_{X}\right), \mathrm{H}^{2}\left(X, \mathcal{O}_{X}\right)$, $\mathrm{H}^{1}\left(X, \mathcal{T}_{X}\right)$ and $\mathrm{H}^{1}\left(X, \mathcal{D}_{X}\right)$ vanish. Then the function tw.quant of the theorem gives a bijection

$$
\text { quant : } \frac{\left\{\text { formal Poisson brackets on } \mathcal{O}_{X}[[\hbar]]\right\}}{\text { gauge equivalence }} \stackrel{\simeq}{\longrightarrow} \frac{\left\{\text { star products on } \mathcal{O}_{X}[[\hbar]]\right\}}{\text { gauge equivalence }} \text {. }
$$

The conditions of Corollary 6.3 are satisfied, for instance, when $X=\mathbf{P}_{\mathbb{K}}^{n}(n$ dimensional projective space over $\mathbb{K}$ ).

Let me finish this survey with a question. Given a variety $X$, with Poisson bracket $\{-,-\}_{1}$ on $\mathcal{O}_{X}$, we can form the Poisson deformation $\mathcal{A}:=\mathcal{O}_{X}[[\hbar]]$ with bracket $\hbar\{-,-\}_{1}$, as in Example 2.2 By viewing $\mathcal{A}$ as a twisted Poisson deformation (cf. Example 5.2), and applying Theorem 6.1. we get a twisted associative deformation $\mathcal{B}:=$ quant $(\mathcal{A})$.

Question 6.4. Does there exist a variety $X$, with a symplectic Poisson bracket $\{-,-\}_{1}$, such that the corresponding twisted associative deformation $\mathcal{B}$ is really twisted?

My feeling is that the answer is positive. And moreover, an example should be when $X$ is any Calabi-Yau surface, and $\{-,-\}_{1}$ is any nonzero Poisson bracket on $X$. It might be possible to settle this question with an explicit (yet very hard) calculation, since there are explicit formulas for the twisted quantization map.

\section{Appendix A. DG Lie Algebras and Deformations}

The first time DG (differential graded) Lie algebras were used to study problems in deformation theory was in the paper [SS] of Schlessinger and Stasheff (1977). In 1986 Deligne (in a letter to Millson, see [GM]) formulated the idea that "in characteristic zero, a deformation problem is controlled by a differential graded Lie algebra, with quasi-isomorphic differential graded Lie algebras giving the same deformation theory". This idea will be explained in this appendix, for associative and Poisson deformations of smooth affine algebraic varieties. More details can be found in the papers [Ye1, Ye5]. 
Recall that a DG Lie algebra is a graded $\mathbb{K}$-module $\mathfrak{g}=\bigoplus_{i \in \mathbb{Z}} \mathfrak{g}^{i}$, with a bracket $[-,-]$ of degree 0 satisfying the graded version of the Lie algebra identities, together with a graded derivation d of degree 1 and square 0 .

Definition A.1. A DG Lie algebra $\mathfrak{g}=\bigoplus_{i \in \mathbb{Z}} \mathfrak{g}^{i}$ is said to be of quantum type if $\mathfrak{g}^{i}=0$ for all $i<-1$.

Given a DG Lie algebra $\mathfrak{g}$, let us define a new DG Lie algebra

$$
\mathfrak{g}[[\hbar]]^{+}:=\bigoplus_{p \in \mathbb{Z}} \hbar \mathfrak{g}^{p}[[\hbar]] \subset \bigoplus_{p \in \mathbb{Z}} \mathfrak{g}^{p}[[\hbar]]
$$

in which $\hbar$ is central.

The Maurer-Cartan equation in $\mathfrak{g}[[\hbar]]^{+}$is

$$
\mathrm{d}(\omega)+\frac{1}{2}[\omega, \omega]=0
$$

for an element

$$
\omega=\sum_{j=1}^{\infty} \omega_{j} \hbar^{j} \in \mathfrak{g}^{1}[[\hbar]]^{+} .
$$

The set of solutions of this equation is denoted by $\operatorname{MC}\left(\mathfrak{g}[[\hbar]]^{+}\right)$.

Let $\exp \left(\mathfrak{g}^{0}[[\hbar]]^{+}\right)$be the pronilpotent group associated to the pronilpotent Lie algebra $\mathfrak{g}^{0}[[\hbar]]^{+}$. There is an action of the group $\exp \left(\mathfrak{g}^{0}[[\hbar]]^{+}\right)$on $\mathfrak{g}^{1}[[\hbar]]^{+}$, and this action preserves the subset $\mathrm{MC}\left(\mathfrak{g}[[\hbar]]^{+}\right)$. One defines

$$
\overline{\mathrm{MC}}\left(\mathfrak{g}[[\hbar]]^{+}\right):=\frac{\mathrm{MC}\left(\mathfrak{g}[[\hbar]]^{+}\right)}{\exp \left(\mathfrak{g}^{0}[[\hbar]]^{+}\right)},
$$

the quotient set for this action.

Let us return to our deformation problem, where $X$ is a smooth algebraic variety over $\mathbb{K}$. Take an affine open set $U \subset X$, and let $C:=\Gamma\left(U, \mathcal{O}_{X}\right)$.

One can show that any Poisson (resp. associative) deformation of $C$ is isomorphic to $C[[\hbar]]$ as $\mathbb{K}[[\hbar]]$-algebra (resp. $\mathbb{K}[[\hbar]]$-module) augmented to $C$. Thus it suffices to understand formal Poisson brackets (resp. star products) on $C[[\hbar]]$.

Let $\mathcal{T}_{C}$ denote the module of derivations of $C$. For $p \geq-1$ define

$$
\mathcal{T}_{\text {poly }}^{p}(C):=\bigwedge_{C}^{p+1} \mathcal{T}_{C}
$$

So $\mathcal{T}_{\text {poly }}^{-1}(C)=C, \mathcal{T}_{\text {poly }}^{0}(C)=\mathcal{T}_{C}$ and $\mathcal{T}_{\text {poly }}^{1}(C)=\bigwedge_{C}^{2} \mathcal{T}_{C}$. The direct sum

$$
\mathcal{T}_{\text {poly }}(C):=\bigoplus_{p \geq-1} \mathcal{T}_{\text {poly }}^{p}(C)
$$

is a DG Lie algebra, called the algebra of poly derivations of $C$. The Lie bracket is the Schouten-Nijenhuis bracket, and the differential is 0 .

An element

$$
\omega=\sum_{j=1}^{\infty} \omega_{j} \hbar^{j} \in \mathcal{T}_{\text {poly }}^{1}(C)[[\hbar]]^{+}
$$

determines a skew-symmetric $\mathbb{K}[[\hbar]]$-bilinear biderivation of $C[[\hbar]]$, namely

$$
\{f, g\}:=\sum_{j=1}^{\infty} \omega_{j}(f, g) \hbar^{j}
$$

for $f, g \in C$. A calculation shows that $\omega$ satisfies the Maurer-Cartan equation if and only if the corresponding biderivation $\{-,-\}$ satisfies the Jacobi identity, i.e. it is a 
formal Poisson bracket on $C[[\hbar]]$. Moreover, the action of group $\exp \left(\mathcal{T}_{\text {poly }}^{0}(C)[[\hbar]]^{+}\right)$ corresponds to gauge equivalences between formal Poisson brackets. In this sense $\mathcal{T}_{\text {poly }}(C)$ controls Poisson deformations of $C$.

The second DG Lie algebra in this picture is that of the poly differential operators. A function $\phi: C^{p+1} \rightarrow C$ is called a poly differential operator of order $\leq i$ if it is a differential operator of order $\leq i$ in each of its arguments. The set of all such operators is denoted by $\mathcal{D}_{\text {poly }}^{p}(C)$. Thus $\mathcal{D}_{\text {poly }}^{-1}(C)=C$ and $\mathcal{D}_{\text {poly }}^{0}(C)=\mathcal{D}(C)$, the ring of differential operators. The direct sum

$$
\mathcal{D}_{\text {poly }}(C):=\bigoplus_{p \geq-1} \mathcal{D}_{\text {poly }}^{p}(C)
$$

is a sub DG Lie algebra of the shifted Hochschild cochain complex (with its Gerstenhaber Lie bracket).

A solution $\omega=\sum_{j=1}^{\infty} \omega_{j} \hbar^{j}$ of the Maurer-Cartan equation in $\mathcal{D}_{\text {poly }}(C)[[\hbar]]^{+}$ determines a star product on $C[[\hbar]]$, by the formula

$$
f \star g:=f g+\sum_{j=1}^{\infty} \omega_{j}(f, g) \hbar^{j}
$$

for $f, g \in C$. Such a star product is called differential (as opposed to an ordinary star product, for which the coefficients $\omega_{j}$ are just $\mathbb{K}$-bilinear; cf. equation (1.1)). The group $\exp \left(\mathcal{D}_{\text {poly }}^{0}(C)[[\hbar]]^{+}\right)$is the group of differential gauge equivalences between differential star products. Thus the $D G$ Lie algebra $\mathcal{D}_{\text {poly }}(C)$ controls differential star products.

Fortunately we have this result:

Theorem A.5 ([Ye5]). Let $C$ be a smooth $\mathbb{K}$-algebra.

(1) Any star product $\star$ on $C[[\hbar]]$ is gauge equivalent to a differential star product $\star^{\prime}$.

(2) Let $\star$ and $\star^{\prime}$ be differential star products on $C[[\hbar]]$, and let $g: \star \rightarrow \star^{\prime}$ be a gauge equivalence. Then $g$ is a differential gauge equivalence.

The proof of Theorem A.5 goes like this: it is well known that star products on $C[[\hbar]]$ are controlled by the shifted Hochschild cochain complex; this was essentially shown already in $\mathbf{G e}$. We know that the inclusion of the DG Lie algebra $\mathcal{D}_{\text {poly }}(C)$ into the shifted Hochschild cochain complex is quasi-isomorphism (cf. [Ye3]). This, together with the Equivalence Theorem (see below), implies part (1) of Theorem A.5 Part (2) is a direct calculation.

The upshot is that the $D G$ Lie algebra $\mathcal{D}_{\text {poly }}(C)$ in fact controls all star products on $C[[\hbar]]$.

Both $\mathcal{T}_{\text {poly }}(C)$ and $\mathcal{D}_{\text {poly }}(C)$ are quantum type DG Lie algebras (this is the reason for the name!).

Geometrically, there are sheaves of DG Lie algebras $\mathcal{T}_{\text {poly }, X}$ and $\mathcal{D}_{\text {poly }, X}$ on $X$, that are quasi-coherent as $\mathcal{O}_{X}$-modules. For any affine open set $U$ as above we have

$$
\Gamma\left(U, \mathcal{T}_{\text {poly }, X}\right)=\mathcal{T}_{\text {poly }}(C),
$$

and likewise for $\mathcal{D}_{\text {poly }}$.

In order to control global deformations one has to resort to some kind of resolution of these sheaves of DG Lie algebras, such as the mixed resolutions mentioned in Theorem 4.3 
Remark A.6. The Hochschild cochain complex is not functorial in $C$ at all. On the other hand the complex $\mathcal{D}_{\text {poly }}(C)$ is functorial with respect to étale homomorphisms $C \rightarrow C^{\prime}$.

Remark A.7. The product $\star$ in equation A.4 could fail to be unital. To ensure that $\star$ has $1 \in C$ as unit, we have to take $\omega \in \operatorname{MC}\left(\mathcal{D}_{\text {poly }}^{\text {nor }}(C)[[\hbar]]^{+}\right)$. Here $\mathcal{D}_{\text {poly }}^{\text {nor }}(C)$ is the algebra of normalized poly differential operators. However, since the inclusion $\mathcal{D}_{\text {poly }}^{\text {nor }}(C) \rightarrow \mathcal{D}_{\text {poly }}(C)$ is a quasi-isomorphism, the sets $\overline{\mathrm{MC}}(-)$ are the same. For this reason we can allow ourselves to neglect the distinction between $\mathcal{D}_{\text {poly }}^{\text {nor }}(C)$ and $\mathcal{D}_{\text {poly }}(C)$ in this survey.

\section{Appendix B. The Universal Quantization Map}

Let $C$ be a smooth $\mathbb{K}$-algebra. There is a canonical homomorphism of complexes of $\mathbb{K}$-modules

given by

$$
\Upsilon_{1}: \mathcal{T}_{\text {poly }}(C) \rightarrow \mathcal{D}_{\text {poly }}(C)
$$

$$
\Upsilon_{1}\left(\partial_{1} \wedge \cdots \wedge \partial_{k}\right)\left(f_{1}, \ldots, f_{k}\right):=\frac{1}{k !} \sum_{\sigma \in S_{k}} \operatorname{sgn}(\sigma) \partial_{\sigma(1)}\left(f_{1}\right) \cdots \partial_{\sigma(k)}\left(f_{k}\right)
$$

for $f_{i} \in C$ and $\partial_{i} \in \mathcal{T}_{C}$. Here $S_{k}$ is the group of permutations. The homomorphism $\Upsilon_{1}$ is called the antisymmetrization map or the HKR map, the latter because of its similarity to the famous Hochschild-Kostant-Rosenberg Theorem. It is known that $\Upsilon_{1}$ is a quasi-isomorphism - see [Ko1] for the $\mathrm{C}^{\infty}$ case, and [Ye1] for the algebraic case - and it induces an isomorphism of graded Lie algebras in cohomology. But $\Upsilon_{1}$ is not a DG Lie algebra homomorphism!

Here is the major result in the area of deformation quantization:

Theorem B.1 (Kontsevich Formality Theorem, [Ko1, 1997). Let $C:=$ $\mathbb{K}\left[t_{1}, \ldots, t_{n}\right]$, the polynomial ring over $\mathbb{K}$, and assume $\mathbb{R} \subset \mathbb{K}$. Then $\Upsilon_{1}$ extends to an $\mathrm{L}_{\infty}$ quasi-isomorphism

$$
\Upsilon=\left\{\Upsilon_{j}\right\}_{j=1}^{\infty}: \mathcal{T}_{\text {poly }}(C) \rightarrow \mathcal{D}_{\text {poly }}(C) .
$$

In other words, $\Upsilon_{1}$ is a DG Lie algebra quasi-isomorphism, up to specified higher homotopies $\Upsilon_{2}, \Upsilon_{3}, \ldots$. Each of the functions $\Upsilon_{j}$ is a poly differential operator, and is invariant under linear change of coordinates in $C$.

There is an induced $\mathbb{K}[[\hbar]]$-multilinear $\mathrm{L}_{\infty}$ quasi-isomorphism

$$
\Upsilon: \mathcal{T}_{\text {poly }}(C)[[\hbar]]^{+} \rightarrow \mathcal{D}_{\text {poly }}(C)[[\hbar]]^{+} .
$$

The next result was known in the nilpotent case, namely for artinian parameter algebras as in Remark 1.4 at least since [Ko1]. The complete case was only proved recently [Ye7, Theorem 0.4].

Theorem B.3 (Equivalence Theorem). Let $\Phi: \mathfrak{g} \rightarrow \mathfrak{h}$ be an $\mathrm{L}_{\infty}$ quasi-isomorphism between DG Lie algebras. Then there is an induced bijection

$$
\overline{\mathrm{MC}}(\Phi): \overline{\mathrm{MC}}\left(\mathfrak{g}[[\hbar]]^{+}\right) \rightarrow \overline{\mathrm{MC}}\left(\mathfrak{h}[[\hbar]]^{+}\right),
$$

functorial in $\Phi$, with an explicit formula.

Combining Theorems B.1 and B.3 we find that there is a canonical bijection

$$
\overline{\mathrm{MC}}(\Upsilon): \overline{\mathrm{MC}}\left(\mathcal{T}_{\text {poly }}(C)[[\hbar]]^{+}\right) \stackrel{\simeq}{\longrightarrow} \overline{\mathrm{MC}}\left(\mathcal{D}_{\text {poly }}(C)[[\hbar]]^{+}\right) .
$$

Therefore: 
Corollary B.4. Assume $\mathbb{R} \subset \mathbb{K}$ and $C=\mathbb{K}\left[t_{1}, \ldots, t_{n}\right]$. Then there is a canonical bijection of sets

$$
\text { quant : } \frac{\{\text { formal Poisson brackets on } C[[\hbar]]\}}{\text { gauge equivalence }} \stackrel{\simeq}{\longrightarrow} \frac{\{\text { star products on } C[[\hbar]]\}}{\text { gauge equivalence }}
$$

preserving first order brackets.

Remark B.5. The reason we require that $\mathbb{K}$ contains $\mathbb{R}$ is because the explicit formula of Kontsevich for the higher homotopies $\Upsilon_{j}$ involves transcendental real numbers. See discussion in Ko2. More recent work (based on ideas of Tamarkin, cf. [CV2]) shows that this requirement can be avoided.

\section{Appendix C. The $\mathrm{L}_{\infty}$ quasi-isomorphism of the Level of Sheaves}

Here is an outline of the proof of Theorem 4.3 Details can be found in the Erratum to [Ye1]. We assume $\mathbb{R} \subset \mathbb{K}$, and $X$ is a smooth $n$-dimensional algebraic variety over $\mathbb{K}$.

A formal coordinate system at a closed point $x \in X$ is an isomorphism of $\mathbb{K}$-algebras

$$
\boldsymbol{k}(x)[[\boldsymbol{t}]]=\boldsymbol{k}(x)\left[\left[t_{1}, \ldots, t_{n}\right]\right] \stackrel{\simeq}{\longrightarrow} \widehat{\mathcal{O}}_{X, x},
$$

where $\boldsymbol{k}(x)$ is the residue field, and $\widehat{\mathcal{O}}_{X, x}$ is the complete local ring.

There is an infinite dimensional scheme Coor $X$, called the coordinate bundle, with a projection $\pi$ : Coor $X \rightarrow X$, which is a moduli space for formal coordinate systems. (In Ko1 the notation for Coor $X$ is $X^{\text {coor }}$.) In particular, for every closed point $x \in X$, the $\boldsymbol{k}(x)$-rational points in the fiber $\pi^{-1}(x)$ stand in bijection to the set of formal coordinate systems at $x$.

To get an idea of how the scheme Coor $X$ looks, let us note that Coor $X=$ $\lim _{\leftarrow} \operatorname{Coor}^{i} X$, where each $\operatorname{Coor}^{i} X$ is the variety parameterizing formal coordinate systems up to order $i$.

Let $\mathcal{P}_{X}$ be the sheaf of principal parts on $X$ (a.k.a. the jet sheaf). Recall that $\mathcal{P}_{X}$ is the formal completion of $\mathcal{O}_{X \times X}$ along the diagonal. As such, $\mathcal{P}_{X}$ is a sheaf of rings on $X$, with two ring homomorphisms $\mathcal{O}_{X} \rightarrow \mathcal{P}_{X}$ (corresponding to the two projections $X \times X \rightarrow X$ ), which make $\mathcal{P}_{X}$ into an $\mathcal{O}_{X}$-bimodule. When we view $\mathcal{P}_{X}$ as a left $\mathcal{O}_{X}$-module, it has the Grothendieck connection

$$
\nabla: \mathcal{P}_{X} \rightarrow \Omega_{X}^{1} \otimes_{\mathcal{O}_{X}} \mathcal{P}_{X},
$$

which is the completion of pullback, under the first projection $X \times X \rightarrow X$, of the standard connection $\mathrm{d}: \mathcal{O}_{X} \rightarrow \Omega_{X}^{1}$. The Grothendieck connection is flat, and its kernel is $\mathcal{O}_{X}$ (coming from the second projection).

Let us denote by $\pi^{*} \mathcal{P}_{X}$ the pullback of the left $\mathcal{O}_{X}$-module $\mathcal{P}_{X}$; and let $\widehat{\pi^{*}} \mathcal{P}_{X}$ be its adic completion. The universal property of $\pi$ : Coor $X \rightarrow X$ implies that there is a canonical isomorphism

$$
\mathcal{O}_{\text {Coor } X} \widehat{\otimes}_{\mathbb{K}} \mathbb{K}[[\boldsymbol{t}]] \cong \widehat{\pi}^{*} \mathcal{P}_{X},
$$

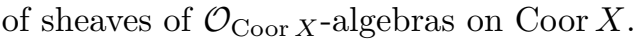

Like (C.1), there are canonical isomorphisms

$$
\mathcal{O}_{\text {Coor } X} \widehat{\otimes}_{\mathbb{K}} \mathcal{T}_{\text {poly }}(\mathbb{K}[[\boldsymbol{t}]]) \cong \pi^{*}\left(\mathcal{P}_{X} \otimes_{\mathcal{O}_{X}} \mathcal{T}_{\text {poly }, X}\right)
$$

and

$$
\mathcal{O}_{\text {Coor } X} \widehat{\otimes}_{\mathbb{K}} \mathcal{D}_{\text {poly }}(\mathbb{K}[[\boldsymbol{t}]]) \cong \pi^{*}\left(\mathcal{P}_{X} \otimes_{\mathcal{O}_{X}} \mathcal{D}_{\text {poly }, X}\right)
$$


of graded Lie algebras on Coor $X$. Let us write $\mathcal{A}:=\Omega_{\text {Coor } X}$, the sheaf of differential forms on Coor $X$; so in particular $\mathcal{A}^{0}=\mathcal{O}_{\text {Coor } X}$. Then, by applying $\mathcal{A} \widehat{\otimes}_{\mathcal{A}^{0}}-$, we get canonical graded Lie algebra isomorphisms

$$
\mathcal{A} \widehat{\otimes}_{\mathbb{K}} \mathcal{T}_{\text {poly }}(\mathbb{K}[[\boldsymbol{t}]]) \cong \mathcal{A} \widehat{\otimes}_{\mathcal{A}^{0}} \pi^{*}\left(\mathcal{P}_{X} \otimes_{\mathcal{O}_{X}} \mathcal{T}_{\text {poly }, X}\right)
$$

and

$$
\mathcal{A} \widehat{\otimes}_{\mathbb{K}} \mathcal{D}_{\text {poly }}(\mathbb{K}[[t]]) \cong \mathcal{A} \widehat{\otimes}_{\mathcal{A}^{0}} \pi^{*}\left(\mathcal{P}_{X} \otimes_{\mathcal{O}_{X}} \mathcal{D}_{\text {poly }, X}\right)
$$

on Coor $X$. However the differentials do not match: to compensate for the Grothendieck connection on the right side, we have to add the differential $\operatorname{ad}(\omega)$ on the left side, where $\omega \in \mathcal{A}^{1} \widehat{\otimes}_{\mathbb{K}} \mathcal{T}_{\text {poly }}^{0}(\mathbb{K}[[\boldsymbol{t}]])$ is a universal MC element.

Due to the Kontsevich Formality Theorem (Theorem B.1) we obtain an induced $\mathcal{A}$-multilinear $\mathrm{L}_{\infty}$ quasi-isomorphism

$$
\Upsilon: \mathcal{A} \widehat{\otimes}_{\mathbb{K}} \mathcal{T}_{\text {poly }}(\mathbb{K}[[\boldsymbol{t}]]) \rightarrow \mathcal{A} \widehat{\otimes}_{\mathbb{K}} \mathcal{D}_{\text {poly }}(\mathbb{K}[[\boldsymbol{t}]])
$$

between sheaves of DG Lie algebras on Coor $X$. (This should be compared to the $\mathrm{L}_{\infty}$ quasi-isomorphism (B.2) in the local case.) Using the isomorphisms (C.2) and (C.3), and twisting $\Upsilon$ by the element $\omega$ (in the sense of [Ye3, Theorem 3.2]), we then obtain an $\mathcal{A}$-multilinear $\mathrm{L}_{\infty}$ quasi-isomorphism

$$
\Upsilon_{\omega}: \mathcal{A} \widehat{\otimes}_{\mathcal{A}^{0}} \pi^{*}\left(\mathcal{P}_{X} \otimes_{\mathcal{O}_{X}} \mathcal{T}_{\text {poly }, X}\right) \rightarrow \mathcal{A} \widehat{\otimes}_{\mathcal{A}^{0}} \pi^{*}\left(\mathcal{P}_{X} \otimes_{\mathcal{O}_{X}} \mathcal{D}_{\text {poly }, X}\right)
$$

If we had a section $\sigma: X \rightarrow$ Coor $X$ then we could pull $\Upsilon_{\omega}$ down to an $\mathrm{L}_{\infty}$ quasi-isomorphism on $X$. However usually there are no global sections of Coor $X$, because of topological obstructions.

The group $\mathrm{GL}_{n}$ acts on Coor $X$ by linear change of coordinates. Let us define LCC $X$ to be the quotient scheme Coor $X / \mathrm{GL}_{n}$. ("LCC" stands for "linear coordinate classes".) So the projection $\pi$ : Coor $X \rightarrow X$ factors through LCC $X$. Recall that the universal quantization of Kontsevich is invariant under linear change of coordinates, namely under the action of the group $\mathrm{GL}_{n}$ and its Lie algebra. This implies that the $\mathrm{L}_{\infty}$ morphism $\Upsilon_{\omega}$ descends to LCC $X$; and hence it suffices to look for sections $\sigma: X \rightarrow \operatorname{LCC} X$ of the projection LCC $X \rightarrow X$.

In the $\mathrm{C}^{\infty}$ context such global sections $\sigma: X \rightarrow \operatorname{LCC} X$ do exists (because the fibers of the bundle LCC $X$ are contractible). But this is not the case in our algebraic situation; so we must use a trick.

Let $G$ be the group of $\mathbb{K}$-algebra automorphisms of $\mathbb{K}[[\boldsymbol{t}]]$. So $G \cong \mathrm{GL}_{n} \ltimes N$, where $N$ is the subgroup of elements that act trivially modulo $(\boldsymbol{t})^{2}$. The group $N$ is pro-unipotent. It turns out that Coor $X$ is a $G$-torsor over $X$, locally trivial in the Zariski topology.

Suppose we are given a finite number of sections

$$
\sigma_{0}, \ldots, \sigma_{q}: U \rightarrow \operatorname{LCC} X
$$

over some open set $U \subset X$. Using an averaging process for unipotent group actions [Ye4, we show that there exists a morphism

$$
\boldsymbol{\sigma}: \boldsymbol{\Delta}_{\mathbb{K}}^{q} \times U \rightarrow \operatorname{LCC} X
$$

which restricts to $\sigma_{j}$ on the $j$-th vertex of $\boldsymbol{\Delta}_{\mathbb{K}}^{q}$. Here $\boldsymbol{\Delta}_{\mathbb{K}}^{q}$ is the $q$-dimensional geometric simplex. 

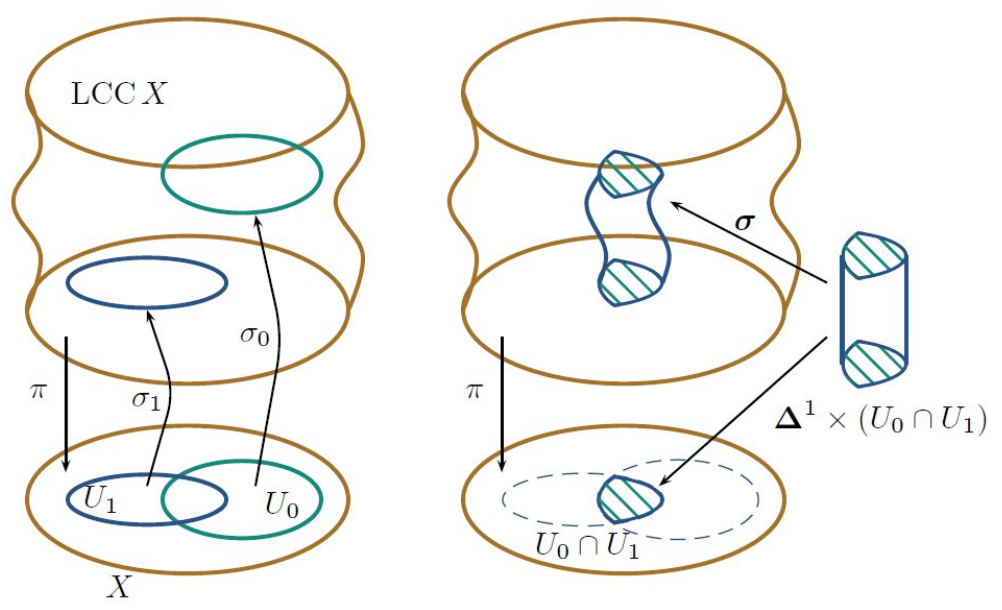

Figure 2. Simplicial sections, $q=1$. We start with sections over two open sets $U_{0}$ and $U_{1}$ in the left diagram; and we pass to a simplicial section $\boldsymbol{\sigma}$ on the right.

Since sections exist locally, we can choose an open covering $\boldsymbol{U}=\left\{U_{i}\right\}$ of $X$, with sections $\sigma_{i}: U_{i} \rightarrow \operatorname{LCC} X$. For any $i_{0}, \ldots, i_{q}$ we then obtain a morphism

$$
\boldsymbol{\sigma}: \boldsymbol{\Delta}_{\mathbb{K}}^{q} \times\left(U_{i_{0}} \cap \cdots \cap U_{i_{q}}\right) \rightarrow \operatorname{LCC} X .
$$

(See Figure 2 for an illustration of the case $q=1$.) As $q$ varies we have a simplicial section of the projection LCC $X \rightarrow X$. Details are in [Ye2].

Another device we use is mixed resolutions. The mixed resolution $\operatorname{Mix}_{U}\left(\mathcal{T}_{\text {poly }, X}\right)$ is a sheaf of DG Lie algebras on $X$, depending on the covering $\boldsymbol{U}$, and equipped with a DG Lie algebra quasi-isomorphism

$$
\mathcal{T}_{\text {poly }, X} \rightarrow \operatorname{Mix}_{U}\left(\mathcal{T}_{\text {poly }, X}\right) .
$$

Likewise for $\mathcal{D}_{\text {poly }, X}$.

The simplicial section $\boldsymbol{\sigma}$ allows us to pull the $\mathrm{L}_{\infty}$ quasi-isomorphism $\Upsilon_{\omega}$ down to $X$, giving an $\mathrm{L}_{\infty}$ quasi-isomorphism

$$
\Psi_{\sigma}: \operatorname{Mix}_{U}\left(\mathcal{T}_{\text {poly }, X}\right) \rightarrow \operatorname{Mix}_{U}\left(\mathcal{D}_{\text {poly }, X}\right)
$$

between these sheaves of DG Lie algebras on $X$. The dependence on $\boldsymbol{U}$ and $\boldsymbol{\sigma}$ disappears when we pass to homotopy classes.

\section{Appendix D. Twisted Deformations and their Gauge Gerbes}

This section summarizes the main concepts of the paper [Ye5.

Recall that a groupoid $\mathrm{G}$ is a category in which all morphisms are invertible. We denote by $\mathrm{G}(i, j)$ the set of arrows from the object $i$ to the object $j$. Note that $\mathrm{G}(i, i)$ is a group. Any element $g \in \mathrm{G}(i, j)$ defines a group isomorphism

$$
\operatorname{Ad}(g): \mathrm{G}(i, i) \stackrel{\simeq}{\longrightarrow} \mathrm{G}(j, j),
$$

namely $\operatorname{Ad}(g)(h):=g \circ h \circ g^{-1}$.

A stack of groupoids $\mathcal{G}$ on $X$ is the geometrization of the notion of groupoid, in the same way that a sheaf of groups is the geometrization of the notion of a 
group. Thus for any open set $U \subset X$ there is a groupoid $\mathcal{G}(U)$. And there are restriction functors $\mathcal{G}(U) \rightarrow \mathcal{G}(V)$ for any inclusion $V \subset U$. These satisfy a rather complicated list of conditions. In particular, given any open set $U \subset X$ and any object $i \in \operatorname{Ob} \mathcal{G}(U)$, there is a sheaf of groups $\mathcal{G}(i, i)$ on $U$. For details see [Ye6. Sections 1-2], or [Gi, BM, KS1.

A stack of groupoids $\mathcal{G}$ is called a gerbe if it is locally nonempty and locally connected.

Definition D.1. Let $X$ be a smooth algebraic variety over $\mathbb{K}$. A twisted associative (resp. Poisson) deformation $\mathcal{A}$ of $\mathcal{O}_{X}$ is the following data:

(1) A gerbe $\mathcal{G}$ on $X$, called the gauge gerbe of $\mathcal{A}$.

(2) For any open set $U \subset X$ and $i \in \mathrm{Ob} \mathcal{G}(U)$, an associative (resp. Poisson) deformation $\mathcal{A}_{i}$ of $\mathcal{O}_{U_{i}}$.

The conditions are:

(a) For any $i \in \operatorname{Ob} \mathcal{G}(U)$, the sheaf of groups $\mathcal{G}(i, i)$ coincides with $\operatorname{IG}\left(\mathcal{A}_{i}\right)$, the sheaf of inner gauge transformations of the deformation $\mathcal{A}_{i}$.

(b) For any $i \in \operatorname{Ob} \mathcal{G}(U)$, any $j \in \operatorname{Ob} \mathcal{G}(V)$, any $W \subset U \cap V$ and any $g \in$ $\mathcal{G}(W)(i, j)$, the isomorphism of sheaves of groups

$$
\operatorname{Ad}(g):\left.\left.\mathcal{G}(i, i)\right|_{W} \stackrel{\simeq}{\longrightarrow} \mathcal{G}(j, j)\right|_{W}
$$

is induced from a (necessarily unique) gauge equivalence

$$
\left.\left.\mathcal{A}_{i}\right|_{W} \stackrel{\simeq}{\longrightarrow} \mathcal{A}_{j}\right|_{W} .
$$

Theorem D.2 ([प्रe6]). Definitions 5.1 and D.1 are equivalent.

The proof relies on the fact that the gauge gerbe $\mathcal{G}$ is pronilpotent, and its abelian slices are coherent $\mathcal{O}_{X}$-modules. Hence for any affine open set $U$ the groupoid $\mathcal{G}(U)$ is nonempty and connected.

Finally let us say a few words on the proof of Theorem 6.1 in Ye5 11

Fix an affine open covering $\boldsymbol{U}=\left\{U_{0}, \ldots, U_{m}\right\}$ of $X$, such that for each $i$ there is an étale morphism $U_{i} \rightarrow \mathbf{A}_{\mathbb{K}}^{n}$. Given a sheaf $\mathcal{G}$ of DG Lie algebras on $X$, the Cech construction gives a cosimplicial DG Lie algebra $\mathrm{C}(\boldsymbol{U}, \mathcal{G})=\left\{\mathrm{C}^{p}(\boldsymbol{U}, \mathcal{G})\right\}_{p \in \mathbb{N}}$. Thus from Theorem 4.3 we deduce that there is a diagram

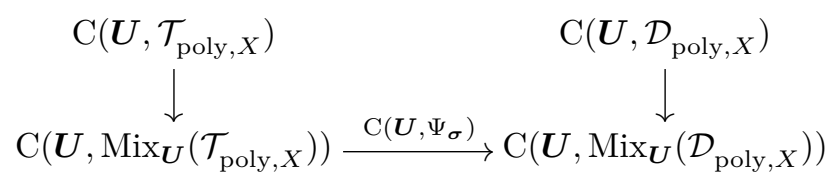

in which the objects are cosimplicial DG Lie algebras, the vertical arrows are cosimplicial DG Lie algebra homomorphisms, and the horizontal arrow is a cosimplicial $\mathrm{L}_{\infty}$ morphism. Moreover, in each cosimplicial dimension $p$ the diagram

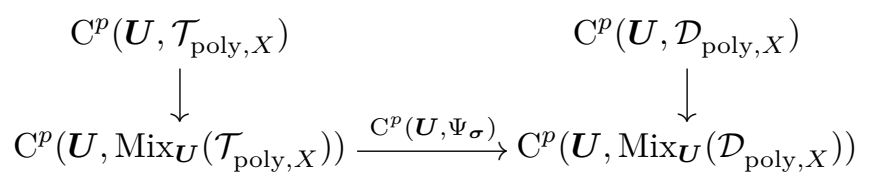

has objects that are quantum type DG Lie algebras, the vertical arrows are DG Lie algebra quasi-isomorphisms, and the horizontal arrow is an $\mathrm{L}_{\infty}$ quasi-isomorphism.

\footnotetext{
${ }^{1}$ This is the proof in the new version of [Ye5], still in preparation as of July 2011.
} 
Now take any quantum type DG Lie algebra $\mathfrak{g}$. To it we associate, in a functorial way, the Deligne 2-groupoid $\operatorname{Del}\left(\mathfrak{g}[[\hbar]]^{+}\right)$, as in $\mathbf{G e}$. If we are given a cosimplicial quantum type DG Lie algebra $\mathfrak{g}=\left\{\mathfrak{g}^{p}\right\}_{p \in \mathbb{N}}$, then there is a cosimplicial 2-groupoid

$$
\operatorname{Del}\left(\mathfrak{g}[[\hbar]]^{+}\right):=\left\{\operatorname{Del}\left(\mathfrak{g}^{p}[[\hbar]]^{+}\right)\right\}_{p \in \mathbb{N}} .
$$

Next let $\mathbf{G}=\left\{\mathbf{G}^{p}\right\}_{p \in \mathbb{N}}$ be any cosimplicial 2-groupoid. To it we associate the set $\operatorname{CDD}(\mathbf{G})$ of combinatorial descent data, and the quotient set by gauge equivalences $\overline{\operatorname{CDD}}(\mathbf{G})$. The sets $\operatorname{CDD}(\mathbf{G})$ and $\overline{\operatorname{CDD}}(\mathbf{G})$ depend functorially on $\mathbf{G}$.

So for a cosimplicial quantum type DG Lie algebra $\mathfrak{g}=\left\{\mathfrak{g}^{p}\right\}_{p \in \mathbb{N}}$ we can associate the set

$$
\operatorname{LDD}\left(\mathfrak{g}[[\hbar]]^{+}\right):=\operatorname{CDD}\left(\operatorname{Del}\left(\mathfrak{g}[[\hbar]]^{+}\right)\right)
$$

of Lie descent data ${ }^{2}$, and its quotient set

$$
\overline{\operatorname{LDD}}\left(\mathfrak{g}[[\hbar]]^{+}\right):=\overline{\operatorname{CDD}}\left(\operatorname{Del}\left(\mathfrak{g}[[\hbar]]^{+}\right)\right) .
$$

See BGNT, Section 3.2], where $\operatorname{LDD}\left(\mathfrak{g}[[\hbar]]^{+}\right)$is called "descent data for Deligne 2 -groupoids".

We have the following theorem, extending [BGNT, Proposition 3.3.1].

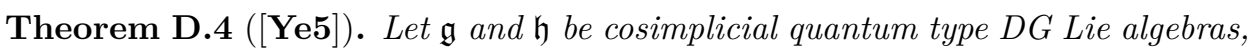
and let $\Phi: \mathfrak{g} \rightarrow \mathfrak{h}$ be a cosimplicial $\mathrm{L}_{\infty}$ quasi-isomorphism. Then there is a bijection

$$
\overline{\operatorname{LDD}}(\Phi): \overline{\operatorname{LDD}}\left(\mathfrak{g}[[\hbar]]^{+}\right) \stackrel{\simeq}{\longrightarrow} \overline{\operatorname{LDD}}\left(\mathfrak{h}[[\hbar]]^{+}\right),
$$

depending functorially on $\Phi$, with an explicit formula.

Applying this theorem to the diagram D.3 we obtain a canonical diagram of bijections of sets

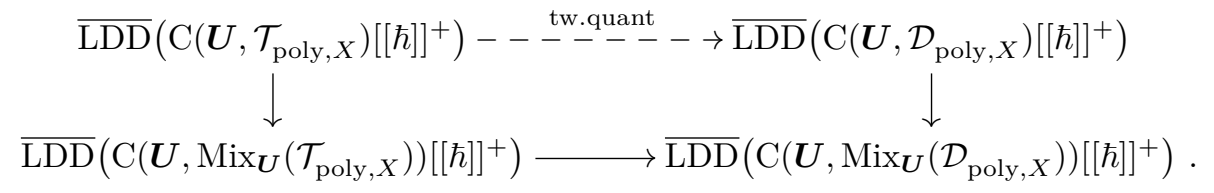

Theorem D.2 implies that twisted Poisson deformations of $\mathcal{O}_{X}$, modulo twisted gauge equivalence, correspond bijectively to elements of

$$
\overline{\operatorname{LDD}}\left(\mathrm{C}\left(\boldsymbol{U}, \mathcal{T}_{\text {poly }, X}\right)[[\hbar]]^{+}\right) .
$$

Likewise, twisted associative deformations, modulo twisted gauge equivalence, correspond bijectively to elements of

$$
\overline{\operatorname{LDD}}\left(\mathrm{C}\left(\boldsymbol{U}, \mathcal{D}_{\text {poly }, X}\right)[[\hbar]]^{+}\right) .
$$

The resulting bijection

$$
\begin{aligned}
\text { tw.quant } & : \frac{\left\{\text { twisted Poisson deformations of } \mathcal{O}_{X}\right\}}{\text { twisted gauge equivalence }} \\
& \stackrel{\simeq}{\longrightarrow} \frac{\left\{\text { twisted associative deformations of } \mathcal{O}_{X}\right\}}{\text { twisted gauge equivalence }}
\end{aligned}
$$

is canonical (independent of $\boldsymbol{U}$ and $\boldsymbol{\sigma}$ ).

\footnotetext{
${ }^{2}$ In the version of [Ye5] dated August 2009, this is called "additive descent data", and denoted by $\operatorname{ADD}\left(\mathfrak{g}[[\hbar]]^{+}\right)$.
} 


\section{References}

BFFLS. F. Bayen, M. Flato, C. Fronsdal, A. Lichnerowicz and D. Sternheimer, Deformation theory and quantization I, II, Ann. Physics 111 (1978), 61-110, 111-151.

BGNT. P. Bressler, A. Gorokhovsky, R. Nest and B. Tsygan, Deformation quantization of gerbes, Adv. Math. 214, Issue 1 (2007), 230-266.

BK. R. Bezrukavnikov and D. Kaledin, Fedosov quantization in algebraic context, Moscow Math. J. 4, no. 3 (2004), 559592.

BS. J. Baez and U. Schreiber, Higher Gauge Theory, in: "Categories in Algebra, Geometry and Mathematical Physics", Contemporary Mathematics 431 (2007), AMS.

BM. L. Breen and W. Messing, Differential geometry of gerbes, Adv. Math. 198 (2005), no. $2,732-846$.

CFT. A. Cattaneo, G. Felder and L. Tomassini, From local to global deformation quantization of Poisson manifolds, Duke Math. J. 115 (2002), no. 2, 329-352.

CKTB. A. Cattaneo, B. Keller, C. Torossian and A. Bruguieres, "Déformation, Quantification, Théory de Lie", Panoramas et Synthèses 20 (2005), Soc. Math. France.

CV1. D. Calaque and M. Van den Bergh, Hochschild cohomology and Atiyah classes, Advances in Mathematics 224 (2010), 1839-1889.

CV2. D. Calaque and M. Van den Bergh, Global formality at the G-infinity level, Mosc. Math. J. 10 (2010), 31-64, 271.

DL. M. De Wilde and P.B.A. Lecomte, Existence of star-products and of formal deformations in Poisson Lie algebra of arbitrary symplectic manifolds, Let. Math. Phys. 7 (1983), 487496.

DP. A. D'Agnolo and P. Polesello, Stacks of twisted modules and integral transforms, in: "Geometric aspects of Dwork theory", Vol. I, II, Walter de Gruyter GmbH and Co. KG, Berlin (2004), 463-507.

Fe. B. Fedosov, A simple geometrical construction of deformation quantization, J. Differential Geom. 40 (1994), no. 2, 21-238.

Gi. J. Giraud, "Cohomologie non abelienne," Grundlehren der Math. Wiss. 179, Springer (1971).

Ge. M. Gerstenhaber, On the deformation of rings and algebras, Ann. of Math. 79 (1964), 59-103.

GK. I.M. Gelfand and D.A. Kazhdan, Some problems of differential geometry and the calculation of cohomologies of Lie algebras of vector fields, Soviet Math. Dokl. 12 (1971), no. 5, 1367-1370.

GM. W.M. Goldman and J.J Millson, The deformation theory of representations of fundamental groups of compact Kahler manifolds, Publ. Math. IHES 67 (1988), 43-96.

GS. V.W. Guillemin and S. Sternberg, "Symplectic Techniques in Physics," Cambridge University Press, Reprint 1990.

Hi. $\quad$ V. Hinich, Descent of Deligne groupoids, Internat. Math. Res. Notices 5 (1997), 223-239.

HY. R. Hübl and A. Yekutieli, Adelic Chern forms and applications, Amer. J. Math. 121 (1999), 797-839.

Ka. M. Kashiwara, Quantization of contact manifolds, Publ. Res. Inst. Math. Sci. 32 no. 1 (1996), 17.

Ko1. M. Kontsevich, Deformation quantization of Poisson manifolds, Lett. Math. Phys. 66 (2003), no. 3, 157-216.

Ko2. M. Kontsevich, Operads and Motives in deformation quantization, Lett. Math. Phys. 48 (1999), 35-72.

Ko3. M. Kontsevich, Deformation quantization of algebraic varieties, Lett. Math. Phys. 56 (2001), no. 3, 271-294.

KS1. M. Kashiwara and P. Schapira, "Categories and Sheaves," Springer, 2006.

KS2. M. Kashiwara and P. Schapira, Deformation quantization modules, arXiv:1003.3304

Lo. W. Lowen, Algebroid prestacks and deformations of ringed spaces, Trans. Amer. Math. Soc. 360 (2008), 1631-1660.

LV. W. Lowen and M. Van den Bergh, Deformation theory of abelian categories, Trans. AMS 358 (2006) no. 12, 5441 - 5483.

Mo. I. Moerdijk, Introduction to the Language of Stacks and Gerbes, eprint math.AG/0212266 at http://arxiv.org. 
NT. R. Nest and B. Tsygan, Deformations of symplectic Lie algebroids, deformations of holomorphic symplectic structures, and index theorems, Asian J. Math. 5 (2001), no. 4, 599-635.

PS. P. Polesello and P. Schapira, Stacks of quantization-deformation modules on complex symplectic manifolds, Intern. Math. Res. Notices. 2004 (2004), 2637-2664.

SS. M. Schlessinger and J. Stasheff, Rational homotopy theory - obstructions and deformations, In Proc. Conf. on Algebraic Topology, Vancouver, pages 7-31, 1977. LMM 673.

VdB. M. Van den Bergh, On global deformation quantization in the algebraic case, J. Algebra 315 (2007), 326-395.

Ye1. A. Yekutieli, Deformation Quantization in Algebraic Geometry, Adv. Math. 198 (2005), 383-432. Erratum: Adv. Math. 217 (2008), 2897-2906.

Ye2. A. Yekutieli, Mixed Resolutions and Simplicial Sections, Israel J. Math. 162 (2007), 1-27.

Ye3. A. Yekutieli, Continuous and Twisted L-infinity Morphisms, J. Pure Appl. Algebra 207 (2006), 575-606.

Ye4. A. Yekutieli, An Averaging Process for Unipotent Group Actions, Representation Theory 10 (2006), 147-157.

Ye5. A. Yekutieli, Twisted Deformation Quantization of Algebraic Varieties, math.AG/0801.3233.

Ye6. A. Yekutieli, Central Extensions of Gerbes, Advances in Mathematics 225 (2010), 445486 .

Ye7. A. Yekutieli, MC Elements in Pronilpotent DG Lie Algebras Eprint arXiv:1103.1035

Department of Mathematics, Ben Gurion University, Be’er Sheva 84105, Israel

E-mail address: amyekut@math.bgu.ac.il 